\title{
The Growing Astronomical Unit and the Dark Energy Influence on the Baryonic Matter Evolution
}

\author{
H. Harutyunian ${ }^{1 *}$, A. Grigoryan ${ }^{1}$ \\ ${ }^{1}$ NAS RA V. Ambartsumian Byurakan Astrophysical Observatory (BAO), Armenia \\ *E-mail: hhayk@bao.sci.am
}

\begin{abstract}
The effect of the Earths removal from the Sun is considered. This effect is difficult to interpret within the traditional paradigm of cosmic objects formation and evolution. Currently tidal mechanism is used as the most appropriate tool for explaining the Earth removal although no any quantitative data exist concerning the deceleration of Suns spin. We argue in favor of the dark energy influence, which, on the other hand, leads to the gradual increase of the Sun mass. Applying self-consistently all the known consequences of the interaction between the dark energy carrier and the baryonic matter, one arrives at a conclusion that the energy of the baryonic objects should grow up which, in its turn, increases their total mass. The mass growth of the Sun is estimated using the relevant observational data.
\end{abstract}

Keywords: Dark energy - expansion at short scales - second law of thermodynamics - energy-mass transformation - mass growth - Astronomical unit - Sun mass.

\section{Introduction}

When the linear relation between the galaxies distance and redshift was discovered in 20s of the last century (Lemaître 1927; Hubble 1929) it was interpreted as a ponderable proof of correctness of the solutions obtained for the Einstein gravitational equation (Friedman 1922; Lemaitre 1927). No attention was paid to the fact that the equation has been solved for the homogeneous and isotropic distribution of the matter in the Universe, while these conditions are more or less satisfied only for the large scales. Although, the inhomogeneity scale was not a common knowledge then. Moreover, at the end of last century the observational base of cosmology changed drastically owing to the discovery of the accelerated expansion of the Universe (Riess et al 1998; Perlmutter et al 1999). Actually, the revealed new behavior of the 
Universe expansion should change scientific views on the Universe structure and evolution. However, the predominant paradigm remained unchanged in its main axioms. The Universe origination due to the unique grand explosion followed by countless local, so called, Kantian condensations of matter in various scales was updated with several fitting parameters, but otherwise it continued serving as the only adoptable and comprehensive doctrine, describing the birth and evolution of everything. On the other hand, observational data constantly provided by ground based and orbital observatories grows with an accelerating rate, and the existing theories continuously need inventive updates of fitting parameters for explaining the variety of phenomena and regularities. Researchers use this kind of tricks for reconciling the dominant paradigm with empirical data if necessary long enough. One can recall a number of such situations in the history of science dictated due to a desperate need of overcoming any deadlock appeared because of discrepancy between new empirical data and established theories. As a striking example of such a situation, one can remind of the inconsistency revealed between very high dispersion of galaxy velocities and the total mass of the clusters. The idea on the existence of dark matter (Zwicky 1933; 1937) was invented then as a fitting tool, to overcome the ideological difficulty. Now, after more than eighty years, one can state that this fitting trick remained a thing in itself, despite the fact that it is used everywhere, where any system of cosmic objects is suspected to have positive total energy. Even worse, such an assumption is now considered a sign of a good scientific manner. According to the predominant concept on the cosmic objects formation, now we have a strange physical picture, where the universe is expanding with an accelerating rate to the infinity, while at the shorter scales everything shrinks, making material objects of bigger and more massive clumps. It does mean that a violation of continuity occurs due to these mutually exclusive processes. Therefore, for the self-consistency of the physical picture one should point out the scales where discontinuity expected or the turning point. This issue is extremely important to consider in detail. Actually, we hold a different point of view. Keeping in mind that the expansion of the Universe in fact is the reflection of processes occurring in the micro world, we try to reveal, wherever possible, all the physical consequences of this physical mechanism, staying at the same time in the frame of the modern physical knowledge. For this approach the analysis of the lunar retreat served as a bench mark (Harutyunian 1995), which showed that one can interpret correctly the Moon removal if the Universe expansion rate is taken into account for the smaller space scales. Some other physical consequences show up when this approach is used, provided, that all the physical laws and regularities are taken into account keeping always in mind the self-consistency of all the physical picture (Harutyunian 2017a; 2017b). In this report we consider the observed change of the Astronomical Unit (AU) again using the hypothesis concerning the universality of the Universe expansion. 


\section{Removal of Earth from Sun}

On the base of analysis of radiometric measurements in the inner Solar System suggested that the secular increase in the unit distance on 154 meters per century (Krasinsky and Brumberg 2004). Calculations show that this increase is much larger than can be accounted for any known mechanism. The trivial one, taking into account the mass loss gives very small effect. Indeed, owing to radiation, it loses about $7 \times 10^{-14} M_{\odot}$ per year. Besides, it due to the mass ejection Sun loses in addition $1.5 \div 2 \times 10^{-14} M_{\odot}$ per year. Thus, our Sun loses yearly less, than about $9 \times 10^{-14} M_{\odot}$. One can calculate easily the upper limit of the AU growth, taking place due to the mass loss of Sun. One needs to write the Earth angular momentum conservation law with the dynamical equilibrium of the Earth keeping the planet in the orbit:

$$
\begin{gathered}
\frac{M_{\oplus} v^{2}}{R}=G \frac{M_{\odot} M_{\oplus}}{R^{2}} \\
M_{\oplus} v R=\text { const }
\end{gathered}
$$

where $R$ is the Astronomical Unit, $M_{\oplus}$ is the Earth mass and $v$ is the orbital velocity of Earth. From (1) and (2) one can find the following relation:

$$
M_{\odot} R=\text { const }
$$

provided that the Earth mass does not change. One finds using (3) that the growth of the Astronomical Unit is not more than 1.35 meter per century, which is on one order of magnitude smaller than the observed value. Hence, the most natural, from the viewpoint of the traditional physics, mechanism cannot explain the observed value of the AU growth. Researchers made several attempts to explain this possible secular increase of AU, including e.g., the effects of the cosmic expansion (Krasinsky and Brumberg 2004; Mashhoon et al. 2007; Arakida 2009), mass loss of the Sun (Krasinsky and Brumberg 2004, Noerdlinger 2008), the time variation of gravitational constant G (Krasinsky and Brumberg 2004), the influence of dark matter (Arakida 2009). Another interpretation, which uses the old idea proposed for the tidal acceleration in the Earth-Moon system, based on the conservation of the total angular momentum appeared about a decade ago (Miura et al 2009). However, if there are methods for estimating the Earths spin decreasing rate for the Earth-Moon system, there is no any applicable method for Suns spin change. Moreover, one cannot solve the paradox of the very rapid retreat of Moon even in the case of the Earth-Moon system, if one stays in the frame of the mentioned traditional approach (Harutyunian 1995). Indeed, according to analysis of the chronography of eclipses observed during last two thousand years, for the deceleration of the Earths diurnal rotation period amounted about 1.8ms per century (Stephenson Morrison 1995; 
Morrison Stephenson 2002). It is not difficult then finding the lunar retreat rate if one considers that the portion of angular momentum lost by Earth completely transfers to the Moon. Evidently, for finding the removal rate one should take into account the angular momentum conservation law with the Kepler third law. Then one finds the following relation:

$$
k_{E} \frac{4 \pi}{5} \frac{M_{\oplus} R_{\oplus}^{2}}{T}+2 \pi \frac{m a^{2}}{p}+k_{M} \frac{4 \pi}{5} \frac{m r^{2}}{p}=\text { const }
$$

where the coefficients $k_{E}$ and $k_{M}$ depend on radial density distribution in the Earth and Moon, correspondingly, $T$ is the period of the Earth diurnal rotation, $m$ is the mass of Moon, $a$ - the average radius of lunar orbit, $r$ - the radius of Moon, $p$ - the period of lunar rotation. In the given relation, the third term is much smaller compared with the first two and therefore can be neglected. Then from the (4) one easily can derive the following formula:

$$
\frac{\Delta a}{a}=0.664 \frac{M_{\oplus}}{m} \frac{R_{\oplus}^{2}}{a} \frac{p}{T} \frac{\Delta T}{T},
$$

or after putting the constants into the relation

$$
\frac{\Delta a}{a}=0.406 \frac{\Delta T}{T} .
$$

For the Earths deceleration rate equal to $1.8 \mathrm{~ms}$ per century, one obtains $3.26 \mathrm{~cm}$ for the lunar annual retreat, which is sufficiently below of the observed value $3.82 \pm 0.07 \mathrm{~cm}$ (Dickey et al, 1994). It does mean that the angular momentum lost by the Earth is not enough to explain the lunar retreat rate. The situation sufficiently changes, if one takes into account the possibility of the Hubble-like expansion at the smaller scales. If one applies the Hubble constant value equal to $70 \mathrm{~km} / \mathrm{s}$ per $\mathrm{Mpc}$, one finds the value of $2.74 \mathrm{~cm}$ per year provided by cosmological expansion solely. This part of lunar retreat does not depend on the angular momentum transfer from the Earth to the Moon. In this framework, the Earth radius also should grow up. Simple calculations made for finding this growth amounts to about $0.455 \mathrm{~mm}$ per year. This increase of the Earth radius decelerates the Earths diurnal rotation by $1.23 \mathrm{~ms}$ per century. Therefore, only a portion of deceleration equal to $0.57 \mathrm{~ms}$ per century remains, which can be caused by tidal effects. The remained portion of angular momentum provides additional rate of lunar removal, which amounts to $1.03 \mathrm{~cm}$ per year. These two effects together result $3.77 \mathrm{~cm}$ growth of the lunar orbit radius per year, which is in the error box of the observed value. This mechanism allows using of some physically valid suggestions as well, which makes it much more flexible for usage. On the other hand, it separates the role of two physical mechanisms and shows that only about 25 percent of the lunar retreat rate can be stipulated for tidal effects. 


\section{Hubble expansion influence on the Astronomical Unit}

Let us stay in the frame of the universality of the Hubble expansion. Then one can calculate the value of its growth solely due to the universal expansion. It is easy to find that for the Hubble constant equal to $70 \mathrm{~km} / \mathrm{s}$ per Mpc the relative growth of the length unit amounts $7.14 \times 10^{-11}$ per year. If the Astronomical Unit obey the same law of expansion, it should increase by $10.7 \mathrm{~m}$ per year, which exceeds the observable value by two orders of magnitude. Therefore, it seems at first glance that one cannot hope for any natural solution of the problem in this frame of thinking. However, the wonderful result obtained for the Earth-Moon system suggests that the main idea likely is plausible, and one needs to find how to use it more correctly. First idea coming to mind about this is the one, which requires finding a physical mechanism preventing the Earth from moving away from the Sun. No doubt, the mass growth of the Sun could prevent the Earths moving away. One of the authors (Harutyunian) has mentioned that the idea of dark energy and its possible influence on the baryonic matter are not considered with proper attention. One can do it from the viewpoint of general physical ideas. First, one should take into attention that dark energy has been discovered thanks to the galaxies acceleration. Then one inevitably arrives at a conclusion that dark energy (or something carrying dark energy) interacts with the ordinary baryonic matter and transfers to the later some energy to accelerate all the baryonic matter. Hence, a regular energy exchange should occur there between the ordinary baryonic matter and the carrier of dark energy, whatever it is. Second, the second law of thermodynamics states, that in the process of interaction between systems of different energies, the system possessing of higher energy loses some part of it, which goes to another one of lower energy. Modern physics considers this law as a monotonic growth of entropy isolated systems. One should bear in mind that the second law of thermodynamics belongs to the class of physical laws, which possess of high priority. According to Eddington the law that entropy always increases holds, I think, the supreme position among the laws of Nature (Eddington 1927). Third, all the objects composed of baryonic matter exist as such exceptionally thanks to negative energy balance (or lack of energy). Gravitationally bound objects possess of negative potential energy, and one can find in any textbook on the general physics the formula describing the energy depending on its mass and size. The atomic nuclei are the natural tools of transforming mass into energy and vice versa. One needs a definite quantity of energy to destroy any atomic nucleus and divide into separate nucleons, increasing by this their combined mass. Moreover, the contemporary physics of elementary particles states that much more energy is needed for destroying the elementary particles and obtaining free 
quarks. Therefore, combining all three statements one arrives at inevitable conclusion that all the objects of the Universe composed of baryonic matter should grow in their mass during the evolution. This conclusion is very important from, at least, two points of view. First, using its mechanism of expansion the Nature uses the expansion energy also for increasing the mass of the baryonic Universe, preventing thus excessive decrease of its density. It seems that the Nature acts according self-consistent scenario programmed in a way to keep the average density unchanged. Second, this mechanism of energy-mass transformation explains the paradox once introduced by the big bang paradigm, which states that the baryonic matter of the Universe was appeared during one act of creation. If one applies physical knowledge in a self-consistent way, one should expect immediate collapse of the newborn universe but not its expansion as one observes at present time. Indeed, it is obvious, that the Universe could not exist if it was born in the same mass it has at present. The baby baryonic Universe, which had many times smaller radius, was within the Schwarzschild radius for long time and should collapse but not expand. It does mean that its mass was many times smaller not to fall into the conditions obeying the requirement of the gravitational collapse. If we agree that the Universe is expanding and it was expanding during all the time, we inevitably should agree that its mass was increasing in course of its expansion.

\section{The rate of solar mass growth}

One can use the relation (3) for estimating the mass growth necessary for compensating the universal expansion rate. Then one should take into attention both effects, namely, the expected growth of the AU without mass change and the shortening of the AU growth with the mass increase:

$$
M_{\odot} R(1+\delta)=M_{\odot}\left(1+\Delta_{M}\right) R\left(1+\Delta_{R}\right),
$$

where $\delta=7.14 \times 10^{-11}$ is the growth of the unit length per year for the Hubble constant amounting $70 \mathrm{~km} / \mathrm{sec}$ per Mpc, $R \Delta_{R}=15 \mathrm{~cm}$ is the observed growth of the AU. It is easy to find that the rate of mass growth for the Sun is equal to

$$
\Delta_{M}=\frac{\delta-\Delta_{R}}{1+\Delta_{R}}=7.04 \times 10^{-11}
$$

Obtained mass growth amounts about $4.5 \times 10^{15} \mathrm{~g} / \mathrm{s}$, which is equivalent to the energetic power of $4.5 \times 10^{36} \mathrm{erg} / \mathrm{s}$ or about 1000 times of the solar luminosity. This is a huge amount, which can explain the discrepancy between the measured and predicted values of the AU growth. Nevertheless, the growth of the mass of Sun, if it occurs, should have a natural physical explanation, and we will dwell on this in a little more detail. To do this, we turn again to the structural features of the baryonic matter. The basis of all 
baryonic objects is the atomic structure, as well as the structural features of the elementary particles, the carriers of mass, charge, and spin of the baryonic substance. It is also obvious that almost all the mass of the substance we observe in the Universe is accumulated in atomic nuclei. Moreover, only atomic nuclei and elementary particles possess of remarkable property responsible for existence of all the objects. That is their self-consistent change of individual masses, depending on their binding degree and other physical conditions, in which they are located. Although it is considered, for example, that all baryons of the same name are indistinguishable and in no way differ from each other, they may have different masses in different atomic nuclei, that is, in different nuclei they transform different parts of their mass into the binding energy. This is a very important empirical fact. Figuratively speaking, this natural fitting mechanism makes baryonic configurations of nuclei energetically balanced with the surrounding physical conditions. Indeed, nuclear binding energy calculated per nucleon changes in a wide range. For the nucleus ${ }^{2} H$ it amounts about $1.112 \mathrm{Mev}$, and increases for ${ }^{3} \mathrm{H}$ up to $2.827 \mathrm{Mev}$, thought the replacing one neutron by a proton decreases the binding energy for ${ }^{2} \mathrm{He}$ down to $2.573 \mathrm{Mev}$, while for the alpha particle ${ }^{4} \mathrm{He}$ it increases up to $7.074 \mathrm{Mev}$. The largest values of the nuclear binding energy have the iron group nuclei, namely, ${ }^{56} \mathrm{Fe},{ }^{58} \mathrm{Fe},{ }^{60} \mathrm{Ni}$ and ${ }^{62} \mathrm{Ni}$, which possess of binding energies $8.791 \mathrm{Mev}, 8.793 \mathrm{Mev}, 8.781 \mathrm{Mev}$ and $8.795 \mathrm{Mev}$, accordingly. One can find many other values of nuclear binding energy showing that the binding energy can take on a variety of values, showing thus the variability of the nucleus mass in a nucleus depending on physical conditions. Taking into account these facts and empirical regularities one arrives at a conclusion that it is not very heretical if one will consider the possibility of changing of the binding energy of the given atomic nuclei over the time due to evolution effects. This conclusion follows from the indisputable facts that baryons in different nuclei can exist with different amounts of mass defect, and that baryonic matter interacts with dark energy carrier. Interaction of various systems of energy carriers obey the thermodynamic laws, including the second law of thermodynamics. If the premises is correct, then one should look more precisely for the physical consequences of such physical effects. Let us emphasize for the later consideration that dark energy evidently has positive sign, since it implements a huge physical work accelerating the Universe expansion. In contrast with it, internal energy of baryonic objects is negative. According to the second law of thermodynamics, the interaction between the baryonic objects and the carrier of dark energy, baryonic objects should gain energy and therefore their internal energy will increase, which does mean decreasing the absolute value of the energy. Gravitationally bound spherical object of mass $M$ and 
radius $R_{U}$ possesses of energy equal to

$$
U=-k_{U} G \frac{M^{2}}{R_{U}},
$$

where the coefficient $k_{U}$ depends on the radial distribution of density in the given object. The gravitational energy increase for such objects should mean decrease of the ratio at the right hand side of the formula (9). It can happen if the radius $R_{U}$ increases more rapidly than $M^{2}$. On the other hand, the baryonic mass of an object grows if the total mass of atoms increases. It can happen due to the interaction between the carrier of dark energy and atomic nuclei, resulting decrease of binding energy and mass defect. This is the plausible way of the mass growth effect for all the objects of the Universe. The suggested mechanism is perfectly new. It assumes that in the course of the Universe evolution all the objects, starting with elementary particles and atomic nuclei and up to gravitationally bound ones undergo a type of secular variation, which is resulted by their interaction with the carrier of dark energy. Thanks to this variation, they gradually change their intrinsic features to be always in the balanced physical interrelation. All the secular variations are caused by the energy interchange or rather energy injection processes, when dark energy carrier gradually exerts influence upon the baryonic objects giving them definite portions of energy. Depending on their intrinsic features, the objects of various hierarchical levels react in completely different ways. Non the less, the general trend for all these objects are several they all increase their gravitational mass, they all can decay in various ways when the internal energy excides some boundary threshold and they transform the portions of dark energy into familiar forms of energy to release it in the form of radiation, mass ejection or other. Within the given context, the change of atomic nuclei can be represented on the base of a phenomenological analysis. If one adopts the law of conservation of baryons in the Universe, then one should arrive at a conclusion, that at the very beginning of our Universe, as we imagine it, baryons possessed only the negligible portion of their masses. Figuratively speaking, there were embryos of baryons packaged into atomic nuclei characterized by enormous mass defect. Nowadays the atomic nuclei continue to gain mass, if the scenario described above is realistic and makes sense. Simply extrapolating this process back to the past, one inevitably concludes, the farther into the past, the fainter baryonic embryos were. Another important conclusion concerns the radioactive nuclei we observe today. The most massive stable nucleus existing today is ${ }^{206} \mathrm{~Pb}$ with its 206 baryons. All other nuclei, which consist of more baryons, are instable. Even ${ }^{209} \mathrm{Bi}$ traditionally regarded as the heaviest nuclei appeared to be alpha radioactive, although its half-life is billion times longer than the estimated age of the Universe. If the secular change of nuclear properties is something real and the nuclear binding energy was bigger in the past, one should consent that there were stable nuclei composed of much 
more baryons in the past.

\section{Concluding remarks}

The contemporary physics provides the only toolkit for interpretation and description of the world consisted of matter. It does mean that one should operate by approved and valid concepts when trying to explain results of experiments or astronomical observations. However, approved by time does not yet guarantee the applied concept is certainly right for all the similar conditions or it was right everywhere it was suggested to be right a priory. We consider here a scenario according which the Hubble expansion is not the prerogative of cosmologic scale only but it is a particular manifestation of the universal expansion taking place at all scales. In other words, we consider the Universe where dark energy implements physical work and interact with the baryonic objects at all scales. Interaction between the dark energy carrier and baryonic objects causes and predetermines the evolutionary path for all baryonic objects in our world. This approach seems to be an extremely heretical one if considered from the conventional viewpoint. One can easily find the roots of such prejudice in relation to any concept speaking in favor of expansion effects at smaller scales. It is hidden in the predominant hypothesis on the formation of cosmic objects - the kantian events at all scales which suggest condensation of matter for reaching the balanced dynamical conditions. Actually the original idea concerning the origin of cosmic objects due to the expansion, decay and fragmentation processes belongs to Viktor Ambartsumian who was insisting for long time on the existence of pre-stellar super-dense matter, which gradually transforms into ordinary matter giving born cosmic objects at different hierarchical levels. The idea could not gain many supporters because the physical laws we apply do not allow existence of such masses in small volumes. Therefore, the idea has been consigned to oblivion. However, the situation drastically changes if one takes into account the physical consequences of the interaction between baryonic matter and dark energy. Any piece of baryonic matter regardless of its size and number of baryons in it accumulates energy and mass growth due to the mentioned interaction, which leads ultimately to the processes of energy release through all the possible mechanisms including the mass ejection and decay of the initial piece. It is evident, that the substance located on or closer to the surface of the decay fragment is more committed to the influence of the external factors and more easily subject to change due to such influences that those parts that are dipper inside. If so, one should expect a negative gradient of the matter evolution or "aging" degree to the center of cosmic objects. The bigger the object, the larger the "age" difference between surface and the center of the object. Therefore, in the depths of massive cosmic objects, baryonic matter must be preserved 
with physical properties it had in the early phases of the Universe evolution. Using the new paradigm on the spatial universality of dark energy, which leads to the expansion effects in the non-quantum world, one can solve the problem appearing in connection with the extremely big masses in small volumes. It follows from the self-consistent application of our knowledge of modern physics. Acting this way one arrives at a conclusion that the selfconsistency of the baryonic world is of a very high level. Interaction with dark energy leads to the growth of spatial sizes of the baryonic world, but also increases its total mass as the injected into the baryonic objects energy transforms into the mass. It does mean, that the matter ejected due to Ambartsumian events could initially have possessed of much less mass and be increased during the ejection process due to the physical conditions change and decrease of the mass defect. In our opinion, this paradigm removes limitations imposed on the Ambartsumian concept on the cosmic objects formation. The fact that the mass of these objects grows in the course of evolution, and a large stock of mass increase is stored in the deep depths of massive space objects in the form of baryon embryos, allows us to solve this problem as well as the paradox already mentioned - why the Universe did not slam immediately after its birth within scenario based on the big bang hypotheses.

\section{References}

Anderson, J.D., Nieto, M.M., 2009, American Astronomical Society, 261, 189, arXiv:0907.2469

Arakida, H., New Astron., 2009, 14, 275

Dickey, J.O., Bender, P.L., Faller, J.E., Newhall, X.X., Ricklefs, R.L., et al, 1994, Science, 265, 482

Eddington, A.S., 1927, The Nature of the Physical World: Gifford Lectures, Cambridge University Press

Friedman, A., 1922, Zeitschrift für Physik, 10 (1), 377. English translation in: Friedman, A. (1999), General Relativity and Gravitation, 31 (12), $1991-2000$.

Harutyunian, H.A., 1995, Astrophysics, 38, 374

Harutyunian, H.A.,2017a, in "Non-Stable Universe: Energetic Resources, Activity Phenomena, and Evolutionary Processes" ASP Conference Series, v.511, 207

Harutyunian, H.A., 2017b, Astrophysics, 60, 627

Hubble, E. P., 1929, Proc. Nat. Acad. Sci. USA ,15, 168

Lemaître, G., 1927, Ann. Soc. Sci. Brux. A 47, 49

Krasinsky, G.A. and Brumberg, V.A., 2004, Celestial Mechanics and Dynamical Astronomy, 90, 267

Mashhoon, B., Mobed, N., Singh, D. 2007, Class. Quant.Grav., 24, 5031. 
Miura, T., Arakida, H., Kasai, M., Kuramata, S., 2009, Publ. Astr. Soc. of Japan, 61, 1247

Morrison, L.V. Stephenson, F.R., 2002, in "Highlights of Astronomy", ed. H.Rickman, 12, 338

Noerdlinger, P. D. 2008, arXiv:0801.3807

Perlmutter, S., Aldering, G., Goldhaber, G., Knop, R.A., Nugent, P., et al, 1999, Ap.J, 517, 565.

Riess, A.G., Filippenko, A.V., Challis, P., Clocchiatti, A., Diercks, P., et al, 1998, AJ, 116, 1009

Stephenson, F.R., Morrison, L.V., 1995, Phil. Trans. R. Soc. London, Ser.A, 351, 165

Zwicky, F., 1933, Helvetica Physica Acta, 6, 110

Zwicky, F., 1937, ApJ, 86, 217 